\section{TOBACCO SMOKE COMPONENTS}

Tobacco smoke components include all substances present in tobacco smoke, irrespective of their origin from tobacco, tobacco additives or atmosphere. Tobacco smoke components are largely identical in mainstream smoke, sidestream smoke and environmental tobacco smoke but their quantitative composition in these different smoke streams varies widely. Most investigations report only the components of mainstream cigarette smoke. First measurements of sidestream smoke components were performed by KEITH and TESH (1). Figure 1 shows that a rather good linear relationship exists between the weight of effluent of mainstream smoke and the number of cigarette puffs $\left(\mathrm{w}_{\mathrm{s}}=50.0 \mathrm{n}\right)$ and that most of the weight of the total effluent (approximately 80\%) consists of gases of atmospheric origin $\left(\mathrm{w}_{\mathrm{a}}=45.0 \mathrm{n}\right)$.

Table 1 presents a material balance for the particle phase and gas phase components of mainstream cigarette smoke (1).

As the mainstream smoke is diluted by air of atmospheric origin, $57.9 \%$ of the mainstream smoke of a cigarette $(289.8 \mathrm{mg}$ according to the conditions described in Table 1) is diluting ambient air, $15.5 \%$ is excess nitrogen, $19.6 \%$ are gas phase components that originate from combustion or pyrolysis and $8.2 \%$ is total particulate matter (TPM). Referring to the data of KEITH and TESH (1), NORMAN (2) gives a quantitative overviewof cigarette mainstream smoke composition which which is shown in Figure 2.

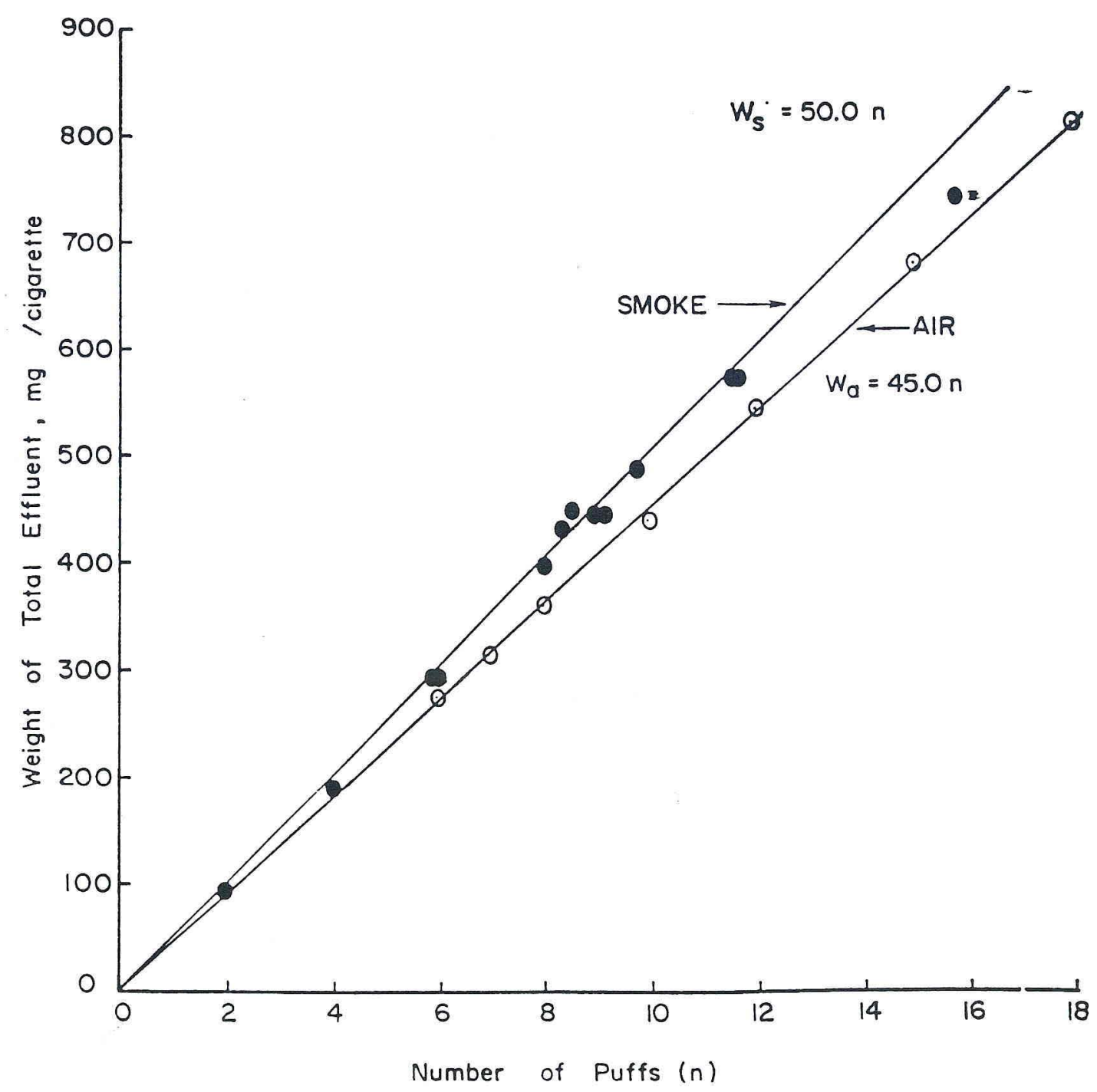

Figure 1.

Weight of effluent as function of number of puffs for cigarette smoke and air (1). 
Table 1.

Material balance of cigarette smoke, $85 \mathrm{~mm}$ unfiltered cigarettes - 10 puffs of $38.9 \mathrm{ml}$ volume, $30 \mathrm{~mm}$ butt length $\left(\mathrm{T}=27^{\circ} \mathrm{C}\right)(1)$.

\begin{tabular}{lrr}
\hline \multicolumn{1}{c|}{ Material } & Weight \\
mg/cig & $\begin{array}{c}\text { Weight } \\
\text { percent of total effluent }\end{array}$ \\
\hline Particulate Matter (inc. cond. $\left.\mathrm{H}_{2} \mathrm{O}\right)$ & 40.6 & 8.2 \\
Nitrogen (67.2 volume \%) & 295.4 & 59.0 \\
Oxygen (13.3 volume \%) & 66.8 & 13.4 \\
Carbon Dioxide (9.8 volume \%) & 68.1 & 13.6 \\
Carbon Monoxide (3.7 volume \%) & 16.2 & 3.2 \\
Hydrogen (2.2 volume \%) & 0.7 & 0.1 \\
Argon (0.8 volume \%) & 5.0 & 1.0 \\
Methane (0.5 volume \%) & 1.3 & 0.3 \\
Water Vapor (R.H. = 0.6) & 5.8 & 1.2 \\
C $_{2}$ - C Hydrocarbons $_{\text {Carbonyls }}$ & 2.5 & 0.5 \\
Hydrogen Cyanide & 1.9 & 0.4 \\
Other Known Gaseous Materials & 0.3 & 0.1 \\
\hline Total & 1.0 & 0.2 \\
\hline Measured Total Effluent & 505.6 & 101.2 \\
\hline
\end{tabular}

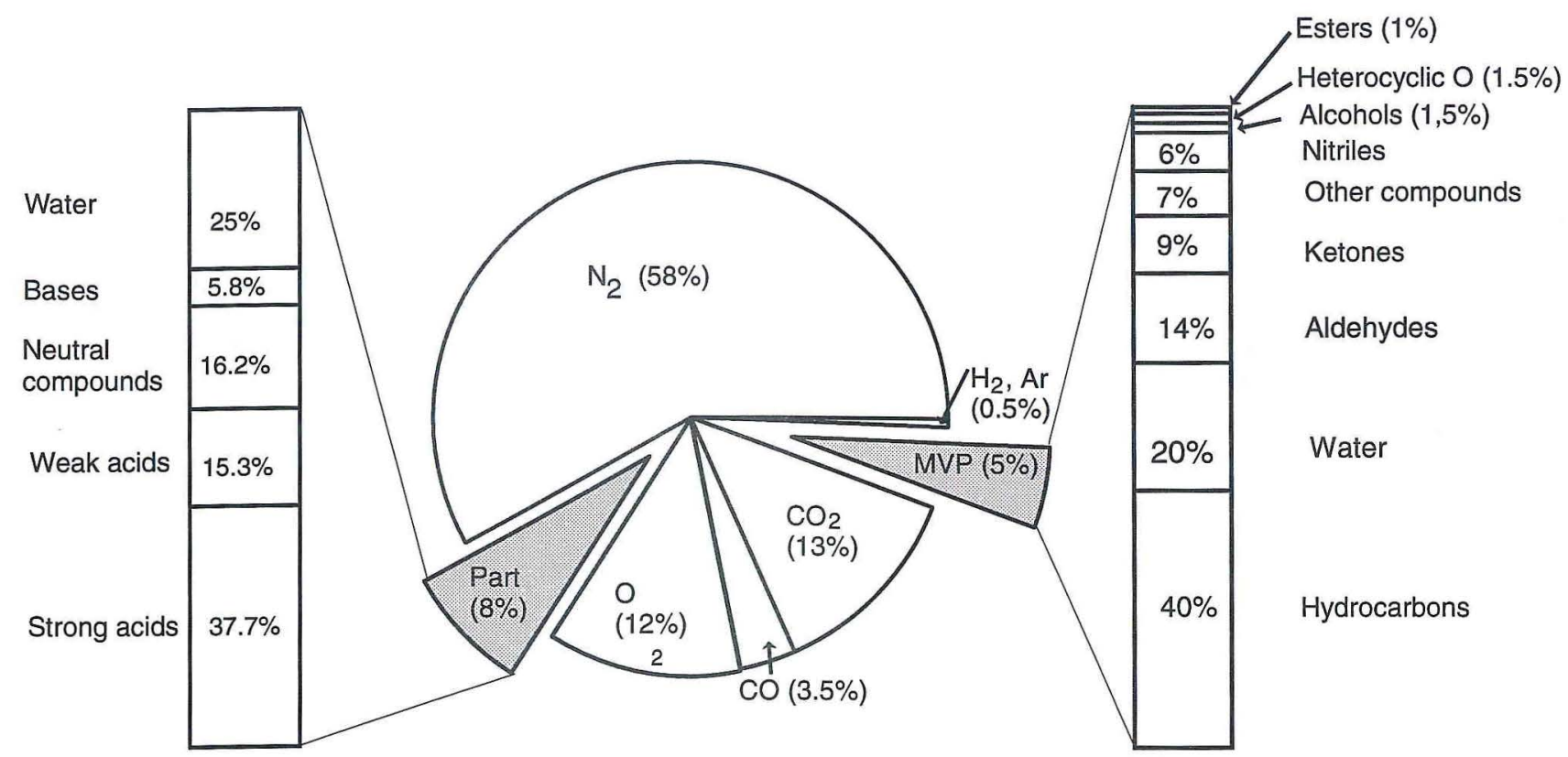

Figure 2.

Approximate chemical composition of mainstream smoke (2). 


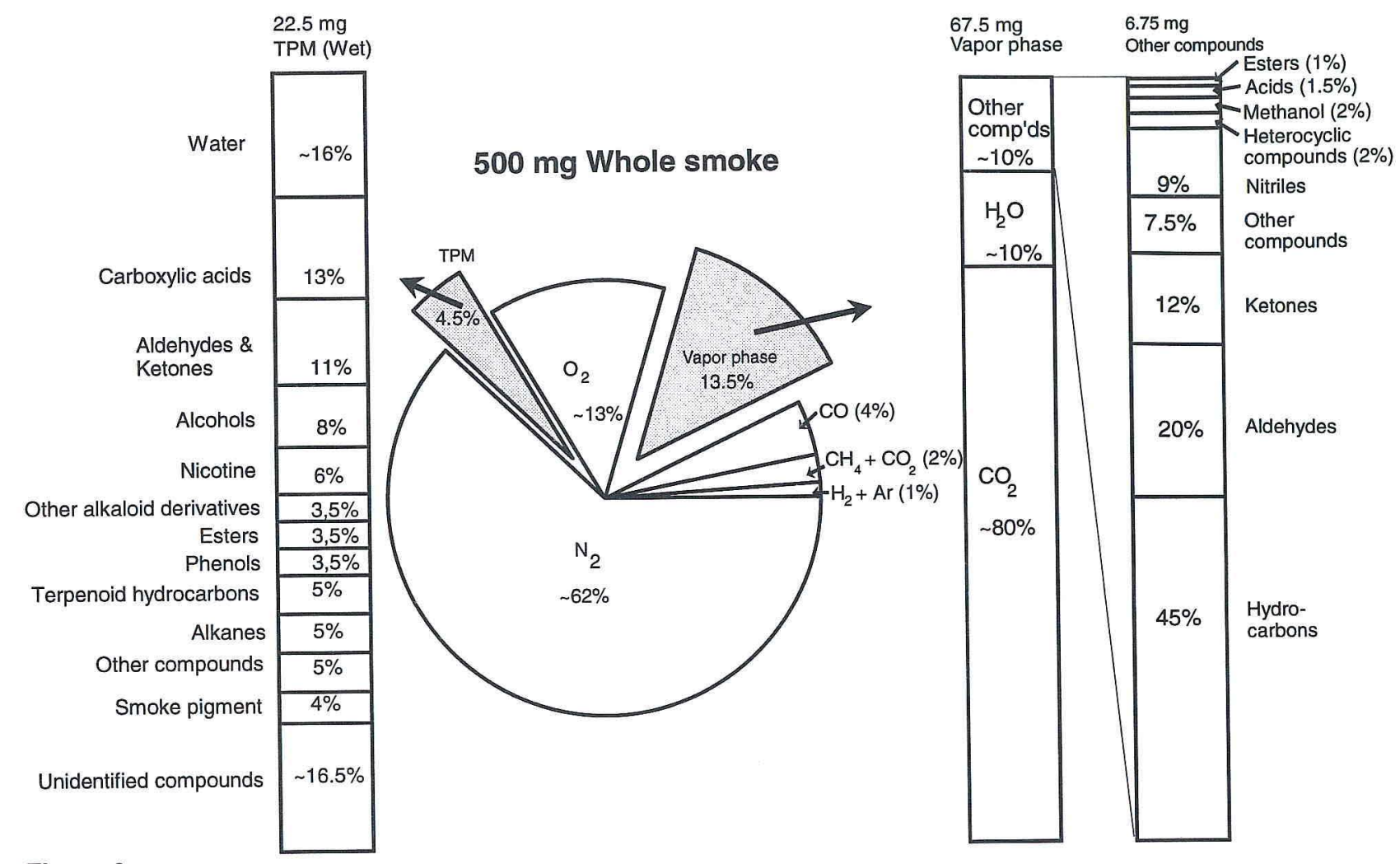

Figure 3.

Composition of cigarette mainstream smoke of an American blend cigarette (3)

NORMAN pays particular attention to the 'particulate phase' ('Part', $8 \%$ ) and the 'miscellaneous vapor phase' ('MVP', $5 \%$ ). These two portions of smoke have been the subject of innumerable studies because of their complex chemical composition and their biological properties. A similar material balance of the composition of mainstream smoke was established by DUBE and GREEN (3) for an unfiltered American blend cigarette of the late 1960's (Figure 3).

The reported percentages of the particulate phase constitutes only $4.5 \%$ of the whole mainstream smoke, and the vapor phase components not of atmospheric origin represent $13.5 \%$ of the total weight. The vapor phase and particulate phase constituents number in the thousands. There have been different approaches to systematically classify these compounds. Table 2 lists different classes of compounds and the number of different components which have been reported in each.

A distribution of the chemical constituents of tobacco smoke present in either the gas phase or particulate phase is reported by GUERIN (4) (Tables 3 - 5).

A further approach is based on classification of the biological acitivity (5). This approach neglects the chemical properties and the degree of toxicity of individual components and is therefore is not very precise. The concentrations of biologically active mainstream smoke components are shown in Table 6.
The amount of tobacco smoke components in mainstream and sidestream cigarette smoke depends on numerous factors such as the tobacco blend, curing, leaf position, cutting width, tobacco additives, cigarette paper, cigarette filter, ventilation.

Table 2.

Approximate number of compounds identified in some major compound classes (3).

\begin{tabular}{lr}
\hline Class & Number \\
\hline Amides, Imides, Lactams & 237 \\
Carboxylic Acids & 227 \\
Lactones & 150 \\
Esters & 474 \\
Aldehydes & 108 \\
Ketones & 521 \\
Alcohols & 379 \\
Phenols & 282 \\
Amines & 196 \\
N-Heterocycles & 921 \\
Hydrocarbons & 755 \\
Nitriles & 106 \\
Anhydrides & 11 \\
Carbohydrates & 42 \\
Ethers & 311 \\
\hline Total & 4720 \\
\hline
\end{tabular}


Table 3 .

Chemical constituents in the gas phase of cigarette smoke (4).

\begin{tabular}{|c|c|c|c|c|}
\hline \multicolumn{5}{|c|}{ Largest representatives } \\
\hline \multicolumn{5}{|c|}{ Quantity } \\
\hline $\begin{array}{c}\text { Type } \\
\text { (minimum number) }\end{array}$ & Compound & $\mathrm{mg} /$ cigarette & $\mu \mathrm{g} /$ cigarette & $\mathrm{ng} /$ cigarette \\
\hline \multirow[t]{10}{*}{ Inorganic gases (15) } & Nitrogen & 295 & & \\
\hline & Oxygen & 67 & & \\
\hline & Carbon dioxide & 68 & & \\
\hline & Carbon monoxide & 16 & & \\
\hline & Argon & 5 & & \\
\hline & Hydrogen & & 700 & \\
\hline & Nitric oxide & & 300 & \\
\hline & Hydrogen cyanide & & 300 & \\
\hline & Hydrogen sulfide & & 90 & \\
\hline & Ammonia & & 100 & \\
\hline Alkanes (17) & Methane & & 800 & \\
\hline \multirow[t]{2}{*}{ Alkenes (41) } & Ethylene & & 160 & \\
\hline & Isoprene & & 400 & \\
\hline Alkynes (5) & Acetylene & & 25 & \\
\hline Cyclic hydrocarbons (12) & Cyclohexane & & & 300 \\
\hline Aromatic hydrocarbons (17) & Toluene & & 80 & \\
\hline Organohalogen (6) & Methyl chloride & & 160 & \\
\hline Alcohols (7) & Methanol & & 180 & \\
\hline \multirow[t]{5}{*}{ Aldehydes (18) } & Acetaldehyde & & 900 & \\
\hline & Formaldehyde & & 30 & \\
\hline & Acrolein & & 70 & \\
\hline & Acetone & & 350 & \\
\hline & Butenone & & 30 & \\
\hline \multirow[t]{2}{*}{ Esters (14) } & Methylformate & & 30 & \\
\hline & Vinylacetate & & & 400 \\
\hline Heterocyclic oxygen (10) & 2-Methylfuran & & 50 & \\
\hline Nitriles (13) & Acetonitrile & & 140 & \\
\hline Nitrosamines (4) & Dimethylnitrosamine & & & 80 \\
\hline Amines (-) & Methylamine & & 4 & \\
\hline \multirow[t]{4}{*}{ Miscellaneous (-) } & Hydrazine & & & 30 \\
\hline & Vinylchloride & & & 25 \\
\hline & Methylnitrate & & & 500 \\
\hline & Water & 6 & & \\
\hline
\end{tabular}


Table 4.

Major constituents of the particulate phase of cigarette smoke (4).

\begin{tabular}{|c|c|}
\hline Chemical & Quantity (mg/cigarette) \\
\hline Water & 6.0 \\
\hline $\begin{array}{l}\text { Humectants } \\
\text { Glycols } \\
\text { Propylene glycol }\end{array}$ & 3.0 \\
\hline $\begin{array}{l}\text { Alkaloids } \\
\text { Nicotine } \\
\text { Nornicotine }\end{array}$ & 1.6 \\
\hline Leaf pigment & 1.5 \\
\hline $\begin{array}{l}\text { Terpenoids } \\
\text { Neophytadiene } \\
\text { Limonene }\end{array}$ & 1.5 \\
\hline $\begin{array}{l}\text { Carboxylic acids } \\
\text { Acetic acid } \\
\text { Palmitic acid }\end{array}$ & 1.2 \\
\hline $\begin{array}{l}\text { Waxes } \\
\qquad \mathrm{nC}_{31}\end{array}$ & 1.2 \\
\hline $\begin{array}{l}\text { Phenols } \\
\text { Phenol } \\
\text { Catechol } \\
\text { Cresols } \\
\text { Hydroquinone }\end{array}$ & 0.6 \\
\hline $\begin{array}{l}\text { Aldehydes } \\
\text { Furfural } \\
\text { Benzaldehyde }\end{array}$ & 0.5 \\
\hline $\begin{array}{l}\text { Phytosterols } \\
\text { Stigmasterol }\end{array}$ & 0.2 \\
\hline
\end{tabular}

\section{REFERENCES}

1. Keith C.H. and P.G. Tesh: Measurement of the total smoke issuing from a burning cigarette; Tob. Sci. 9 (1965) 61-64.

2. Norman V: An overview of the vapor phase, semivolatile and nonvolatile components of cigarette smoke; Tob. Sci. 3 (1977) 28-58

3. Dube, M.F. and C.R. Green: Methods of collection of smoke for analytical purposes; Recent Adv. Tob. Sci. 8 (1982) 42-102.
Table 5.

Trace constituents of the particulate phase of cigarette smoke (4).

\begin{tabular}{lr}
\multicolumn{1}{c}{ Chemical } & \\
& Quantity $(\mu \mathrm{g} /$ cigarette $)$ \\
\hline Aromatic hydrocarbons & \\
Naphthalene & 3 \\
Phenanthrene & 0.4 \\
Pyrene & 0.1 \\
Fluoranthene & 0.1 \\
Benzo[a]pyrene & 0.02 \\
Nitrogenous aromatics & \\
Indole & 15 \\
Pyridine & 40 \\
Quinoline & 2 \\
Anilines & 0.2 \\
Napthylamines & 0.005 \\
Nitrosamines & 1.5 \\
Nitrosonornicotine & 0.5 \\
Nitrosoanatabine & 1.5 \\
Trace elements & \\
Nickel & 0.1 \\
Cadmium & 0.1 \\
\hline
\end{tabular}

4. Guerin, M.R.: Chemical Composition of Cigarette Smoke; in Banbury Report, No. 3: A Save Cigarette, edited by G.B. Gori and F.G. Bock, Cold Spring Harbor Laboratory, Cold Spring Harbor, N.Y., 1980, pp 191-213.

5. Surgeon General Smoking and Health; U.S. Department of Health, Education, and Welfare, 1979.

6. IARC Monographs: Tobacco Smoking; Vol. 38, Lyon: International Agency for Research on Cancer, Lyon, 1986. 
Table 6.

Concentrations of biologically active agents in nonfilter cigarette mainstream smoke (6)

\begin{tabular}{|c|c|}
\hline Smoke constituent & Concentration/cigarette \\
\hline Total particulate matter & $15-40 \mathrm{mg}$ \\
\hline Carbon monoxide & $10-23 \mathrm{mg}$ \\
\hline Nicotine & $1.0-2.3 \mathrm{mg}$ \\
\hline Acetaldehyde & $0.5-1.2 \mathrm{mg}$ \\
\hline Acetic acid & $0.1-1.0 \mathrm{mg}$ \\
\hline Acetone & $100-250 \mu g$ \\
\hline Methanol & $90-180 \mu g$ \\
\hline Nitrogen oxides & $100-600 \mu \mathrm{g}$ \\
\hline Formic acid & $80-600 \mu g$ \\
\hline Hydrogen cyanide & $400-500 \mu g$ \\
\hline Hydroquinone & $110-300 \mu \mathrm{g}$ \\
\hline Catechol & $100-360 \mu \mathrm{g}$ \\
\hline Ammonia & $50-130 \mu g$ \\
\hline Benzene & $20-50 \mu g$ \\
\hline Acrolein & $60-100 \mu g$ \\
\hline Phenol & $60-140 \mu g$ \\
\hline Croton aldehyde & $10-20 \mu \mathrm{g}$ \\
\hline Formaldehyde & $70-100 \mu g$ \\
\hline Pyridine & $16-40 \mu g$ \\
\hline 3-Methylpyridine & $20-36 \mu g$ \\
\hline 2-Cresol & $14-30 \mu \mathrm{g}$ \\
\hline 3- and 4-Cresol & $40-80 \mu g$ \\
\hline 3- and 4-Methylcatechol & $31-45 \mu \mathrm{g}$ \\
\hline Carbazole & $1 \mu \mathrm{g}$ \\
\hline 2-Nitropropane & $0.2-2.2 \mu \mathrm{g}$ \\
\hline N-Nitrosonornicotine & $200-3000$ ng \\
\hline 4-(Methylnitrosamino)-1-(3-pyridyl)-1-butanone & $80-770$ ng \\
\hline N-Nitrosoanabasine & $0-150 \mathrm{ng}$ \\
\hline N-Nitrosodiethanolamine & $0-36$ ng \\
\hline N-Nitrosopyrrolidine & $0-110 \mathrm{ng}$ \\
\hline N-Nitrosodimethylamine & 2 - 20 ng \\
\hline N-Nitrosomethylethylamine & $0-2.7 \mathrm{ng}$ \\
\hline N-Nitrosodiethylamine & $0-2.8 \mathrm{ng}$ \\
\hline$N$-Nitrosodi- $n$-propylamine & $0-1 \mathrm{ng}$ \\
\hline$N$-Nitrosodi- $n$-butylamine & $0-3 \mathrm{ng}$ \\
\hline N-Nitrosopiperidine & $0-9 \mathrm{ng}$ \\
\hline Hydrazine & $32-43 n g$ \\
\hline Urethane & $20-38 \mathrm{ng}$ \\
\hline Vinyl chloride & $1.3-16 \mathrm{ng}$ \\
\hline Benz[a]anthracene & $20-70 \mathrm{ng}$ \\
\hline Benzo[b]fluoranthene & $4-22 n g$ \\
\hline Benzo[j]fluoranthene & $6-21 \mathrm{ng}$ \\
\hline Benzo[k]fluoranthene & $6-12 \mathrm{ng}$ \\
\hline Benzo[a]pyrene & $20-40 \mathrm{ng}$ \\
\hline Dibenz $[a, h]$ anthracene & $4 \mathrm{ng}$ \\
\hline Dibenzo[a,i]pyrene & $1.7-3.2 \mathrm{ng}$ \\
\hline Indeno[1,2,3-cd]pyrene & $4-20 n g$ \\
\hline 5-Methylchrysene & $0.6 \mathrm{ng}$ \\
\hline Dibenz $[a, 1]$ acridine & $2.7 \mathrm{ng}$ \\
\hline Dibenz $[a, h]$ acridine & $0.1 \mathrm{ng}$ \\
\hline 7 H-Dibenzo $[c, g]$ carbazole & $0.7 \mathrm{ng}$ \\
\hline 2-Naphthylamine & $1.7-22 \mathrm{ng}$ \\
\hline 4-Aminobiphenyl & $2.4-4.6 n g$ \\
\hline ortho-Toluidine & $32-160 \mathrm{ng}$ \\
\hline Maleic anhydride & Present \\
\hline 2,3-Dimethylmaleic anhydride & Present \\
\hline Succinic anhydride & Present \\
\hline Coumarin & Present \\
\hline
\end{tabular}

